\section{Isolated and unexplained dilation of the common bile duct on computed tomography scans}

\author{
Naveen B. Krishna, Pavan Tummala, \\ Christopher D. Mehan, Amith V. Reddy, \\ Jeremy A. Hartman, Mohit Mehra, \\ Banke Agarwal \\ Division of Gastroenterology and \\ Hepatology, Saint Louis University \\ School of Medicine, St. Louis, MO, USA
}

\begin{abstract}
Isolated dilation of common bile duct (CBD) (with normal sized pancreatic duct) and without identifiable stones or mass lesion (unexplained) is frequently encountered by computed tomography/magnetic resonance imaging. We studied the final diagnoses in these patients and tried to elucidate factors that can predict a malignant etiology. This is a retrospective analysis of prospective database from a University based clinical practice (20022008). We included 107 consecutive patients who underwent endoscopic ultrasound (EUS) for evaluation of isolated and unexplained CBD dilation noted on contrast computed tomography scans. EUS examination was performed using a radial echoendoscope followed by a linear echoechoendoscope, if a focal mass lesion was identified. Fine-needle aspirates were assessed immediately by an attending cytopathologist. Main outcome measurements included i) prevalence of neoplasms, CBD stones and chronic pancreatitis and ii) performance characteristics of EUS/EUS-fine needle aspiration (EUS-FNA). A malignant neoplasm was found in 16 patients (14.9\%) of the these patients. Even though the presence of obstructive jaundice (ObJ), abnormal liver function tests (LFTs) and intrahepatic biliary dilation (IHD) are used to guide further diagnostic evaluation of these patients, there is limited supporting data.

Based on CT/MRI scans, it is possible to divide patients with dilated CBD into two subsets: those with concomitant dilation of PD (double duct sign) and those without dilated PD (isolated CBD dilation). Published data suggests that the double duct sign is highly suggestive of a malignant etiology. ${ }^{4,5}$ However, the subset of patients with isolated dilation of CBD has not been adequately characterized since earlier studies evaluating patient with dilated CBD did not separate or exclude patients with PD dilation.

In this manuscript, we studied the endoscopic ultrasound (EUS) findings and diagnoses in patients with isolated and unexplained CBD dilation. We also evaluated factors that could potentially predict malignant obstruction including the presence of $0 \mathrm{bJ}$, abnormal LFTs and IHD. Additionally we determined the performance of EUS-fine needle aspiration (EUS-FNA) for identifying malignancy in this cohort of patients.
\end{abstract} study subjects, all with obstructive jaundice (ObJ). Six patients had CBD stones; three with ObJ and three with abnormal liver function tests. EUS findings suggestive of chronic pancreatitis were identified in 27 patients. EUSFNA had $97.3 \%$ accuracy (94.1\% in subset with ObJ) with a sensitivity of $81.2 \%$ and specificity of $100 \%$ for diagnosing malignancy. Presence of $\mathrm{ObJ}$ and older patient age were only significant predictors of malignancy in our cohort. Amongst patients with isolated and unexplained dilation of CBD, the risk of malignancy is significantly higher in older patients presenting with ObJ. EUS-FNA can diagnose malignancy in these patients with high accuracy besides identifying other potential etiologies including missed CBD stones and chronic pancreatitis.

\section{Introduction}

Isolated dilation of common bile duct (CBD) (with normal sized pancreatic duct, PD) and without an identifiable mass lesion or CBD stones (unexplained) is frequently noted on computed tomography (CT) and magnetic resonance imaging (MRI) scans. Further diagnostic evaluation of patients with dilated CBD is performed to look for obstructive lesions including a neoplasm and stones. However, dilation of the CBD can occur without an identifiable obstructive lesion in asymptomatic individuals. $^{1-3}$ In order to optimize management, it is important to know the prevalence of clinically significant obstructive lesions in

\section{Materials and Methods}

This is a retrospective analysis of our prospective database of patients who underwent EUS/EUS-FNA. The database was started in March 2002 and is maintained prospectively. Follow-up information is rigorously collected for quality assurance in our clinical practice. It comprises periodic phone calls to patients, in addition to monitoring correspondence from the referring physicians and primary care physicians, operative notes, surgical pathology and imaging reports. Patients without evidence of cancer based on EUS/EUS-FNA

\section{Study design and patient selection}

Correspondence: Banke Agarwal, Division of Gastroenterology and Hepatology, Saint Louis University School of Medicine, 3635 Vista Avenue, FDT 9S, St. Louis, M0 63110, USA.

Tel. +1.314.577.8764 - Fax: +1.314.577.8757.

E-mail: agarwalb@slu.edu

Key words: dilated common bile duct, malignancy, EUS, intrahepatic biliary dilation, obstructive jaundice, liver function tests.

Contributions: NBK, PT, data collection, interpretation and manuscript preparation; CDM, data collection, analysis and interpretation; AVR, JAH, data collection and analysis; MM, data analysis and interpretation, manuscript preparation; BA, conceived the study, data interpretation and manuscript preparation.

Conflict of interests: the authors report no poten tial conflict of interests.

Received for publication: 24 August 2011.

Revision received: 26 March 2012.

Accepted for publication: 13 April 2012.

This work is licensed under a Creative Commons Attribution NonCommercial 3.0 License (CC BYNC 3.0).

(C) Copyright N.B. Krishna et al., 2012 Licensee PAGEPress, Italy

Gastroenterology Insights 2012; 4:e15

doi:10.4081/gi.2012.e15

undergo repeat imaging by EUS or CT/MRI in 3,6 and/or 12 months (as deemed necessary clinically).

All patients who underwent EUS/EUS-FNA between March 2002 and April 2008 for evaluation of a dilated CBD seen on intravenous contrast CT were considered for inclusion. The CBD was considered dilated if the diameter was $\geq 7 \mathrm{~mm}$ in size ( $\geq 9 \mathrm{~mm}$ in patients with cholecystectomy). ${ }^{6}$ Patients with concomitant dilation of the PD on CT/MRI scans or on EUS exam were not included $(n=131)$. Patients with identifiable CBD stones or mass lesion on CT/MRI were excluded $(n=163)$. Patients with serum bilirubin $>1.30 \mathrm{mg} / \mathrm{dL}$ (predominantly conjugated) were considered to have $0 \mathrm{bJ}$ and to have abnormal LFTs if they had elevated serum alkaline phosphatase with or without an elevation of serum aminotransferases. The study was approved by the Institutional Review Board of the Saint Louis University School of Medicine.

\section{Endoscopic ultrasound examination}

EUS examination was initially performed using radial echoendoscope (EUM-130 and EUM-160 Olympus, Melville, NY). Whenever a suspicious mass lesion was identified on radial EUS, FNA was performed using linear 
echoendoscopes (FG-32A or FG-36A Pentax, Orangeburg, NY or GF-UC140 Olympus, Melville, NY). Multiple FNA passes (1-7 passes) were made using the Echo-tip EUS-FNA needle (Wilson Cook, Winston-Salem, NC) until the cytologist could make a preliminary diagnosis. The cytology specimens were stained by the Diff-Quik and Papanicoulou method (Pap smears) and assessed immediately by a cytologist (Figure 1). A sample was also collected for cell blocks. The final cytologic diagnosis was based on examination of the Pap smears and the cell blocks using standard cytologic criteria. ${ }^{7}$ Special cytology stains were used as indicated to diagnose neuroendocrine tumor or confirm lymphoid tissue. Chronic pancreatitis was diagnosed based on the presence of $\geq 5$ EUS criteria based on Rosemont classification: hyperechoic foci, hyperechoic strands, hyperechoic lobules or foci, accentuation of lobular pattern, irregular gland margin or increased size, cyst, irregular duct contour, visible side branches, hyperechoic duct margin, dilated main duct and stone..$^{8,9}$

\section{Radiologic imaging}

To best address this frequently encountered clinical dilemma, the study was based on data actually used in patient management. CT findings were from the imaging that was performed prior to EUS exam and included those that prompted the referral for EUS. The CT scans had been performed with helical scanners with intravenous contrast but not with pancreatic protocol. The CT scan findings were those reported in the radiology reports and the scans were not reviewed by designated radiologist(s) for purposes of this study. In patients who had endoscopic retrograde cholangio-pancreatography (ERCP) with biliary stent placement, the presence of IHD was sought in the CT scan performed before ERCP. Prominence of central intrahepatic biliary tree was not considered to be IHD.

\section{Follow up and final diagnosis}

Final diagnosis was based on surgical pathology, definitive cytology and/or clinical follow-up of at least 12 months. The median follow-up was 17 months (range 12- 48 months). If the cytologic diagnosis was reported as atypical cells $(\mathrm{n}=10)$, then for purpose of calculating the performance characteristics of EUS-FNA, these patients' cytologic diagnosis was counted as negative for malignancy.

\section{Statistical analysis}

SPSS statistical software (SPSS 17.0; Inc., Chicago, IL) was used for analysis. Chi-square test and Wald's statistics were used to test significance of observed differences. All analyses were two-tailed and statistical significance was accepted as $\mathrm{P}<0.05$. Multivariate logistic regression analysis was done evaluating patient age, associated symptoms, size of CBD and presence of IHD on the probability of malignancy in patients with dilated CBD and ObJ. The Odds ratio estimates, $95 \%$ confidence intervals and corresponding P-values were calculated for significant predictors.

\section{Results}

\section{Patient characteristics}

Tablel summarizes the characteristics of 107 study patients with dilated CBD on CT scan. Their mean age $( \pm$ SD) was $62.7 \pm 14.4$ years. Recent onset ObJ was present in 51 patients and the mean serum bilirubin level prior to stent placement was $5.8 \pm 6.9 \mathrm{mg} / \mathrm{dL}$. A history of abdominal pain was present in 76 patients. Significant weight loss $\geq 10 \mathrm{lbs}$. was present in 15 patients.

The mean diameter of the CBD was $11.5 \pm 2.5 \mathrm{~mm}$ in patients with $0 \mathrm{bJ}, 12.1 \pm 3.5$ $\mathrm{mm}$ in patients with abnormal LFTs, and $10.0 \pm 3.2 \mathrm{~mm}$ in patients with normal LFTs. The PD was normal in size in all patients. Twenty-two patients had IHD on CT, and 29 patients had a cholecystectomy.

\section{Endoscopic ultrasound findings and diagnoses based on presence of obstructive jaundice and abnormal liver function tests}

Figure 2 summarizes the EUS findings and diagnoses in study patients. Amongst 51 patients with ObJ, a focal mass lesion was noted in 29 patients of which 13 were diagnosed malignant by EUS-FNA (Figure 3B). The malignant neoplasms included: 5 pancreatic adenocarcinomas in the uncinate process, 4
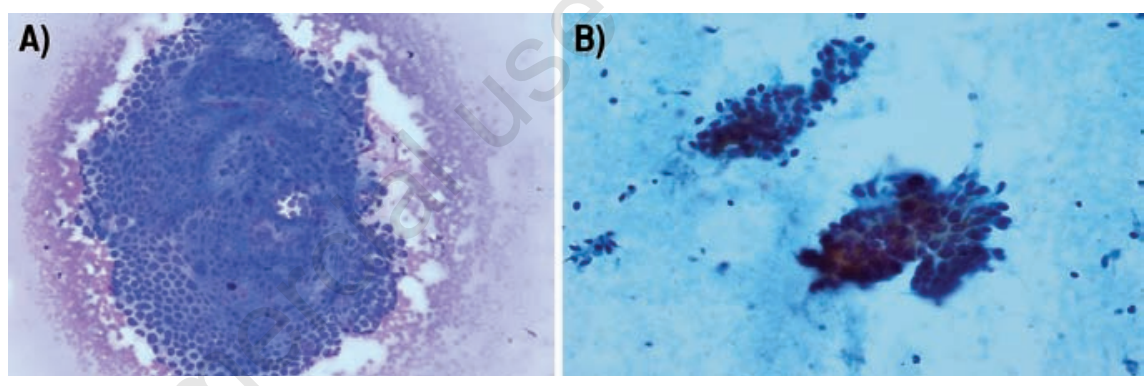

Figure 1. Photomicrographs of pancreatic endoscopic ultrasound/endoscopic ultrasound fine needle aspiration specimens from a patient with pancreatic mass that was diagnosed to be an adenocarcinoma. The smears were stained by Diff-quilk method (A) and by Papanicoulou method (B).

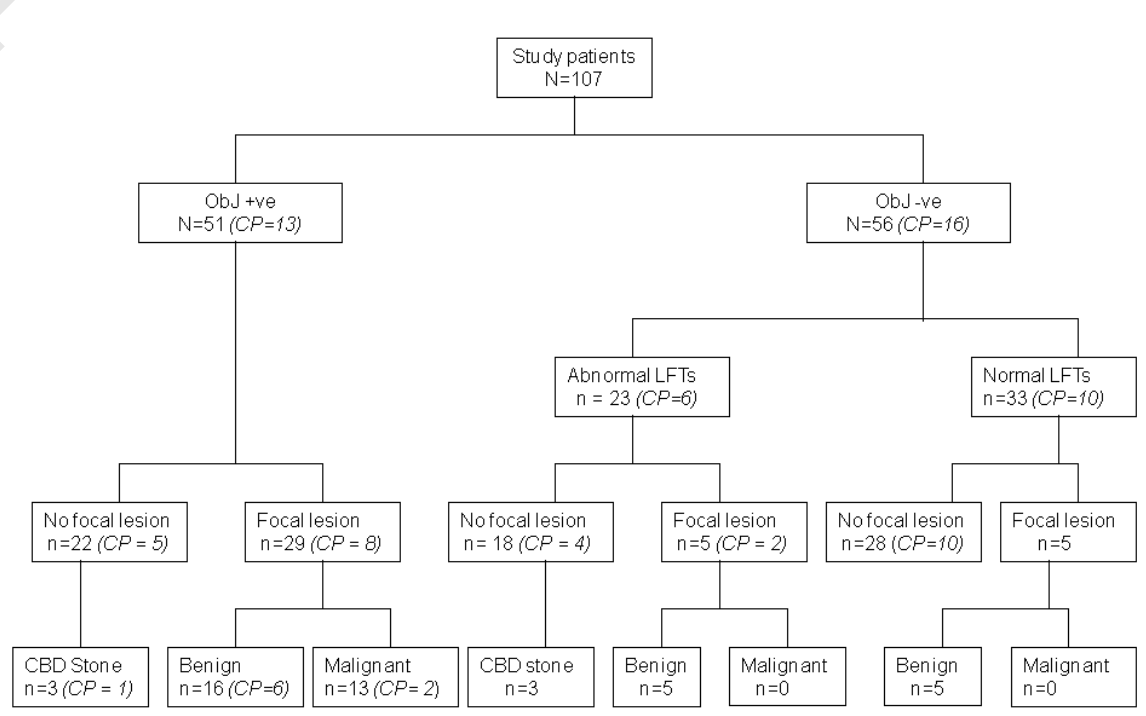

$C P=$ Chronic pancreatitis

Figure 2. Summary of endoscopic ultrasound findings and diagnoses in study patients. 
cholangiocarcinomas, 1 pancreatic lymphoma, 1 plasma cell neoplasm, 1 gallbladder cancer and 1 neuroendocrine tumor. In the remaining 16 patients, FNA cytology revealed benign pancreatic tissue. In three patients, previously missed impacted CBD stones were noted by EUS. These stones were removed by ERCP. One patient had an ampullary adenoma noted by EUS and diagnosed by endoscopic biopsies taken after biliary sphincterotomy with ERCP. EUS evidence of chronic pancreatitis was present in 13 patients; ERCP identified a biliary stricture in 11 of them; remaining two had impacted CBD stone $(n=1)$ and ampullary stenosis $(n=1)$. One of the patients with chronic pancreatitis and biliary stricture had pancreatic adenocarcinoma $(\mathrm{n}=1)$. In 22 of 51 patients with ObJ, EUS and ERCP identified no biliary obstruction.

In the 56 patients without ObJ, EUS identified chronic pancreatitis in 6 of 23 with abnormal LFTs and 10 of 33 patients with normal LFTs. Ten patients ( 5 with abnormal LFTs) had a benign focal non-cystic hypoechoic lesion on EUS examination. Two of the five patients with abnormal LFTs had chronic pancreatitis; one had ampullary adenoma; and the other two yielded normal pancreatic acinar cells. EUS identified previously unnoticed CBD stones in three patients with abnormal LFTs (removed by ERCP). Sixteen patients with abnormal LFTs and 12 patients with normal LFTs also underwent an ERCP and none had a biliary stricture. Ampullary stenosis was diagnosed by ERCP in 2 patients.

\section{Final diagnoses and performance characteristics of endoscopic ultrasound in the study patients}

The final diagnosis in study patients are summarized in Table 2. A malignant neoplasm was found in 16 patients (14.9\% 95\% CI 9.3, 23.0), all with ObJ. Six patients had CBD stones; three with $\mathrm{ObJ}$ and three with abnormal LFTs. EUS findings suggestive of chronic pancreatitis were identified in 27 patients. The performance characteristics of EUS and/or EUS-FNA for diagnosing malignancy are summarized in Table 3 . EUS-FNA had $97.3 \%$ accuracy $(94.1 \%$ in subset with $0 b J)$ with a sensitivity of $81.2 \%$ and specificity of $100 \%$ for diagnosing malignancy. There were three patients with false negative diagnoses for malignancy, all in patients with ObJ. One patient had no identifiable focal mass lesion but had severe chronic pancreatitis on EUS examination and also had a CBD stricture on ERCP. In the second patient, EUS examination revealed a hypoechoic focal lesion around the biliary stent but cytologic diagnosis of malignancy could not be made. The third patient had a $2 \mathrm{~cm}$ mass lesion in relation to the mid-CBD but the cytology was negative for malignancy. All three were diag- nosed to be malignant during surgery. There were no false positive diagnoses.

\section{Predictors of significant pathology} and malignancy in patients with dilated common bile duct

Table 4 summarizes the final diagnoses based on the presence of IHD in study patients with or without ObJ. Amongst patients with ObJ, a malignant obstructive lesion was present in 4 of 11 patients with IHD and 12 of 40 patients without IHD. Only 1 of 6 patients found to have CBD stones had IHD.

Since only patients with $\mathrm{ObJ}$ were finally diagnosed to have malignancy in our cohort, we evaluated the influence of potential factors associated with increased probability of malignancy in patients in this subgroup. We used multivariate logistic regression analysis to evaluate patient age, recent history of significant weight loss ( $\geq 10 \mathrm{lbs}$ in 3 months), history of abdominal pain, CBD diameter and presence of IHD (Table 5). Patient age and CBD diameter were used as continuous variable for the purposes of analysis. Only advancing patient age had significant correlation (HR=1.08 95\% CI 1.00,1.17; $\mathrm{P}=0.03$ ) with the probability of malignancy in our cohort.
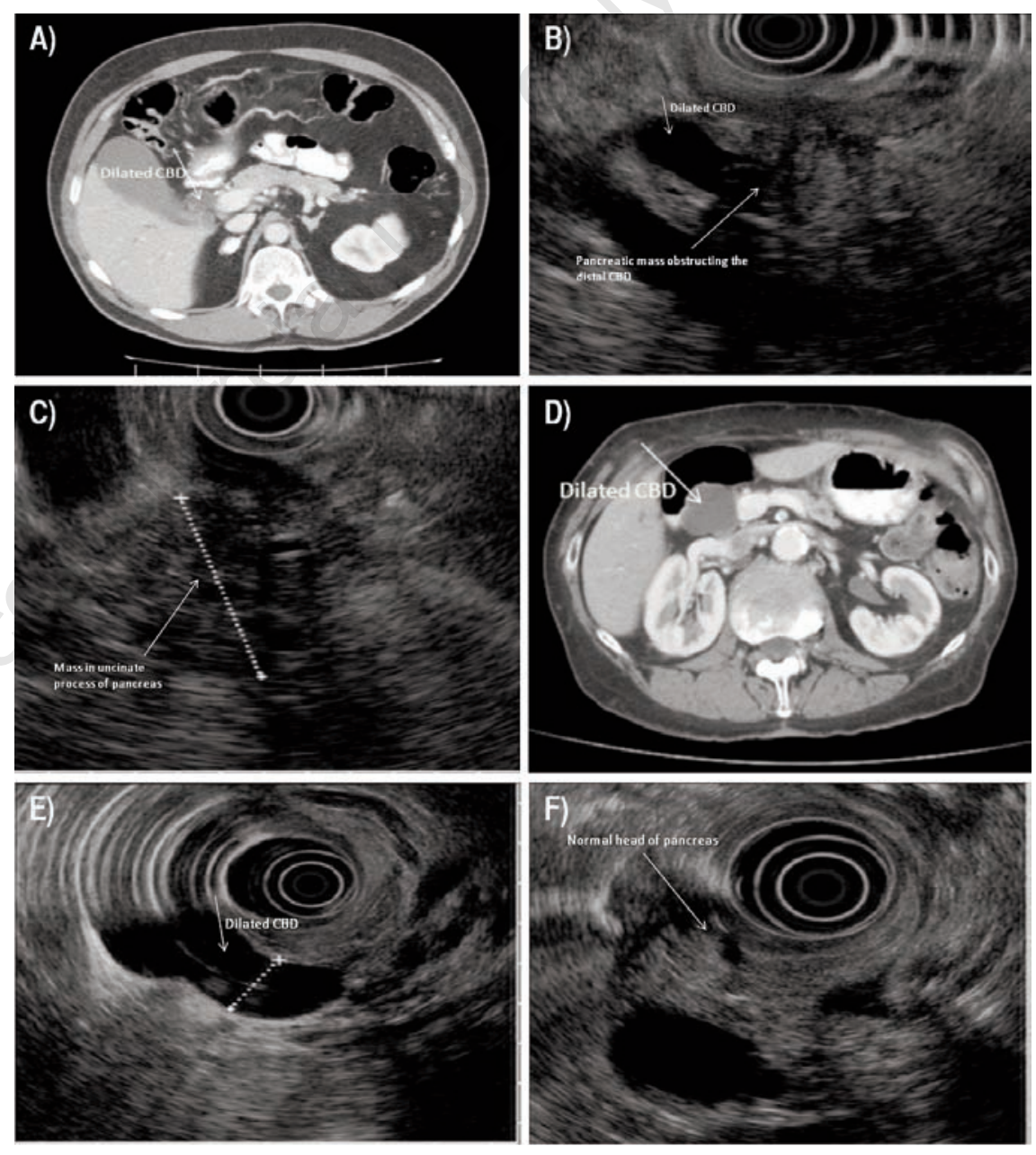

Figure 3. A patient with obstructive jaundice and dilated common bile duct on computed tomography scan. Pancreatic duct is not dilated and a mass lesion is not identifiable on computed tomography scan (A). Endoscopic ultrasound images showing dilated common bile duct terminating into a mass lesion in the pancreas $(B)$ and another view of the same mass arising from the uncinate process $(\mathrm{C})$; patient with abnormal liver function test $s$ but without elevated serum bilirubin and dilated common bile duct noted on computed tomography scan (D). Endoscopic ultrasound identified markedly dilated common bile duct $(\mathrm{E})$ but no obstructive lesions were noted and the pancreatic head appeared normal (F). 


\section{Discussion}

In this manuscript, we assessed the EUS and final diagnoses in patients with radiologic finding of unexplained (without an identifiable mass lesion or stones) and isolated (without associated PD dilation) dilation of the CBD on CT scans. In our cohort, chronic pancreatitis was the most common pathology. Only patients who presented with $0 \mathrm{bJ}$ were found to have a malignant obstructive lesion. Previously unidentified CBD stones were noted in 6 patients (3 in patients with ObJ and 3 in patients with abnormal LFTs). EUS was highly accurate with high negative predictive value for diagnosing malignancy. Besides ObJ, patient age was the only statistically significant predictor of malignancy.

Dancygier et al. had reported on the utility of EUS in patients with extrahepatic biliary obstruction. ${ }^{10}$ Their cohort included patients with dilated CBD and ObJ including those with an identifiable mass or stones on abdominal ultrasound (US) and did not exclude those with associated dilation of PD (radiologic double duct sign). Due to widespread use of CT scans, an important patient subgroup has now emerged comprising of patients with dilation of CBD without PD dilation and without identifiable mass lesion or CBD stones. There is insufficient published data to guide further diagnostic evaluation and management of these patients. EUS is increasingly being used in their evaluation but there is limited published data on the final diagnoses, EUS findings and diagnostic yield, and the performance characteristics of EUS for diagnosing malignancy these patients. These data, if available will allow judicious use of EUS for their diagnostic evaluation.

Songur et al. reported that in a cohort of 90 patients with dilated CBD on US with or without abnormal LFTs and no identifiable etiology, 12 patients had a malignant etiology and all with 0bJ. ${ }^{3}$ However, it is not possible to determine the proportion of ObJ patients who had a malignant diagnosis in their cohort. They also did not exclude patients with double duct sign. We found that in our cohort of patients with isolated and unexplained dilation of CBD and ObJ, the prevalence of malignant etiology was 31.4\% (95\% CI-18.6, 44.1), markedly lower than the reported prevalence of malignancy (58-100\%) in patients with double duct sign. Due to a very high prevalence of malignancy in patients with double duct sign, surgical exploration is often recommended for further management unless there is an obviously unresectable mass lesion on CT/MRI scans; however, in patients with isolated CBD dilation, further diagnostic evaluation to look for malignancy seems more appropriate in view of comparatively lower prevalence of malignancy.
EUS-FNA was highly accurate for diagnosing malignancy and also identified previously unrecognized impacted CBD stones in three patients in our cohort. EUS may potentially have an important role in further evaluation of these patients.

In contrast to patients with $0 \mathrm{bJ}$, the prevalence of malignancy in patients with unexplained dilation of CBD without ObJ seems much lower in published studies. Avni et al. reported finding two patients with periampullary carcinoma in their small cohort of 14 patients with unexplained CBD dilation $\geq 15$ $\mathrm{mm}$ and without $0 \mathrm{bJ} .{ }^{11}$ Malik et al. evaluated 47 patients with dilated CBD noted on US, 32 with normal LFTs and 15 with abnormal LFTs but without clinical jaundice (serum bilirubin $<3.5 \mathrm{mg} / \mathrm{dL}$ ). ${ }^{2}$ They reported a single case of ampullary tumor in their cohort, in a patient with abnormal LFTs. However, it is not clear from their manuscript if this patient had subclinical jaundice with serum bilirubin between
1.0 and $3.5 \mathrm{mg} / \mathrm{dL}$, and if the ampullary tumor was malignant. Kim et al. evaluated 77 patients with dilated CBD without ObJ and found 1 patient with adenocarcinoma and 1 patient with tubular adenoma of the ampulla. ${ }^{1}$ Both these patients had normal LFTs. In all three studies, patients with concomitant dilation of PD (double duct sign) were not specifically excluded and it was also not mentioned if the patients with adenocarcinoma had concomitant PD dilation. Several studies have reported a very high prevalence of malignancy in patients with double duct sign and it is therefore important to distinguish these patients from those with isolated dilation of CBD. ${ }^{4,5}$ In our cohort of 56 patients with isolated and unexplained dilation of CBD and without ObJ, none were found to have a malignant etiology. The number of patients in these studies, including the present one is relatively small. However, even if the data from all these studies are pooled despite the above-mentioned

Table 1. Patient characteristics.

\begin{tabular}{|c|c|c|c|c|}
\hline & $\begin{array}{l}\text { Obstructive } \\
\text { Jaundice } \\
\mathrm{N}=51(\%)\end{array}$ & $\begin{array}{r}\text { No jau } \\
\text { N=56 } \\
\text { Abnormal LFT } \\
\text { N=23(\%) }\end{array}$ & $\begin{array}{l}\text { ndice } \\
\text { i(\%) } \\
\text { Normal LFT } \\
\text { N=33(\%) }\end{array}$ & $\begin{array}{c}\text { Overall } \\
\mathrm{N}=107(\%)\end{array}$ \\
\hline Age (years) & $64.4 \pm 16.2$ & $61.5 \pm 11.2$ & $60.9 \pm 13.5$ & $62.7 \pm 14.4$ \\
\hline $\begin{array}{l}\text { Gender } \\
\text { Male } \\
\text { Female }\end{array}$ & $\begin{array}{l}22(20.6) \\
29(27.1)\end{array}$ & $\begin{array}{c}6(5.6) \\
17(15.8)\end{array}$ & $\begin{array}{c}6(5.6) \\
30(25.2)\end{array}$ & $\begin{array}{l}34(31.8) \\
73(68.2)\end{array}$ \\
\hline $\begin{array}{l}\text { Associated symptoms } \\
\text { Abdominal pain } \\
\text { Weight loss } \geq 10 \mathrm{lbs} \text {. } \\
\text { Mean diameter of the CBD (mm) } \\
\text { Intrahepatic ductal dilation } \\
\text { H/o cholecystectomy }\end{array}$ & $\begin{array}{c}41(38.3) \\
9(8.4) \\
11.5 \pm 2.5 \\
11(10.3) \\
14(13.1)\end{array}$ & $\begin{array}{c}13(12.1) \\
1(0.9) \\
12.1 \pm 3.5 \\
4(3.7) \\
7(6.5)\end{array}$ & $\begin{array}{c}22(20.5) \\
5(4.6) \\
10.0 \pm 3.2 \\
7(6.5) \\
8(7.4)\end{array}$ & $\begin{array}{l}76(71.0) \\
15(14.0) \\
11.2 \pm 3.0 \\
21(19.6) \\
29(27.1)\end{array}$ \\
\hline
\end{tabular}

LFT, liver function test; CBD, common bile duct.

Table 2. Final diagnoses in study patients.

\begin{tabular}{lcccc} 
& $\begin{array}{c}\text { No jaundice } \\
\text { Nastructive } \\
\text { Jaundice } \\
\text { N=51(\%) }\end{array}$ & $\begin{array}{c}\text { Abnormal LFT } \\
\text { N=23(\%) }\end{array}$ & $\begin{array}{c}\text { Normal LFT } \\
\text { N=33(\%) }\end{array}$ & $\begin{array}{c}\text { Overall } \\
\text { N=107(\%) }\end{array}$ \\
Benign (n=91) & & & & \\
No identifiable obstructive lesion & $19(17.8)$ & $13(12.1)$ & $21(19.6)$ & $53(49.5)$ \\
Chronic pancreatitis & $11(10.3)$ & $6(5.6)$ & $10(9.3)$ & $27(25.2)$ \\
CBD stone & $3(2.8)$ & $3(2.8)$ & - & $6(5.6)$ \\
Ampullary Stenosis & $1(0.9)$ & - & $2(1.8)$ & $3(2.8)$ \\
Ampullary adenoma & $1(0.9)$ & $1(0.9)$ & - & $2(1.9)$ \\
Malignant (n=16) & & & & \\
Adenocarcinoma of pancreas & $6(5.6)$ & - & - & $6(5.6)$ \\
(2 CP in the background) & $6(5.6)$ & - & - & $6(5.6)$ \\
Cholangiocarcinoma & $1(0.9)$ & - & - & $1(0.9)$ \\
Neuroendocrine tumor & $1(0.9)$ & - & - & $1(0.9)$ \\
Plasma cell neoplasm & $1(0.9)$ & - & - & $1(0.9)$ \\
Malignant lymphoma & $1(0.9)$ & - & & \\
Ca gallbladder & & & & - \\
\hline
\end{tabular}

LFT, liver function test; CBD, common bile duct. 
limitations, the prevalence of malignancy in patients with dilated CBD with or without abnormal LFTs is markedly lower than in those with $0 \mathrm{bJ}$.

In our cohort, the presence of IHD did not correlate with a higher prevalence of significant pathology especially malignancy or CBD stones. This seems counterintuitive especially since it is well known that progressive IHD develops with longstanding biliary obstruction including that due to malignant tumors. We believe that our findings reflect that with increasing access to ERCP and EUS, more and more patients nowadays undergo diagnostic evaluation in the early stages of biliary obstruction before significant IHD occurs. In addition, idiopathic IHD can happen, as noted in our cohort. Amongst patients with dilated CBD and ObJ, older age was the only variable that was significantly associated with the probability of malignancy. Presence of symptoms (abdominal pain and weight loss), presence of IHD or larger size of the CBD did not seem to predict a malignant diagnosis in our cohort. Future studies need to further evaluate these factors for their predictive value for significant pathology including malignancy to justify continuing their use in clinical decision making.

The present study has limitations inherent to its retrospective design and the present data are therefore not definitive. Whether biliary dilation occurs following cholecystectomy and how much dilation is pathologic is still debated even though several studies use a larger size cutoff in patients with $\mathrm{h} / \mathrm{o}$ cholecystectomy. ${ }^{12-14}$ We similarly used a larger size cutoff in patients who were post-cholecystectomy. Chronic pancreatitis was diagnosed in our cohort by EUS based on previously published criteria which are widely accepted though they have never been validated against surgical pathology. ${ }^{15,16}$ From our data, it seems that chronic pancreatitis may be an important cause of isolated CBD dilation in non-jaundiced patients without an obstructing mass lesion or CBD stones. Another potential limitation is that sphincter of Oddi manometry was not performed in patients without an identifiable obstructive lesion by EUS. Sphincter of Oddi dysfunction is a well known cause of biliary dilation and likely was the etiology in at least some of our study patients. These patients, especially those with history of abdominal pain may possibly benefit from $\mathrm{ERCP} \pm$ sphincter manometry.

The strength of the study is that it is based on data actually used in clinical management. A review of CT scans by dedicated academic radiologists specializing in body imaging potentially would have identified a mass lesion or CBD stone in a few more study patients, but in real life most CT scans are interpreted by community radiologists as was the case with patients in the present study. Another potential strength is that the study addresses a clinical scenario that is being encountered more and more frequently in contemporary clinical practice. Even though, previous studies have addressed patients with CBD dilation, they did not separate the patients with double duct sign from those with isolated CBD dilation, which have markedly different prevalence of malignancy. This limits their clinical applicability. The present data are therefore timely and can potentially help in making management deci- sions in patients with isolated and unexplained dilation of CBD even though they need confirmation in future studies.

\section{Conclusions}

To conclude, in patients with an isolated and unexplained dilation of CBD noted on CT, the risk of malignant obstruction is markedly high-

Table 3. Performance characteristics of endoscopic ultrasound/endoscopic ultrasoundfine needle aspiration for diagnosing malignancy.

\begin{tabular}{lccc} 
& \multicolumn{2}{c}{ Obstructive jaundice } & \\
& Yes & No & Overall \\
& $\mathrm{N}=51(\%)$ & $\mathrm{N}=56(\%)$ & $\mathbf{N}=107(\%)$ \\
True positive & $13(12.1)$ & 0 & $13(12.1)$ \\
False positive & 0 & 0 & 0 \\
\hline False negative & $3(2.8)$ & 0 & $3(2.8)$ \\
True negative & $35(32.7)$ & $56(52.3)$ & $91(85.0)$ \\
\hline Sensitivity \% & $81.3 \%$ & - & $81.3 \%$ \\
(95\% confidence interval) & $(62.1,100.0)$ & & $(62.1,100.0)$ \\
Specificity & $100 \%$ & $100 \%$ & $100 \%$ \\
\hline Positive predictive value & $100 \%$ & - & $100 \%$ \\
Negative predictive value & $92.1 \%$ & $100 \%$ & $96.9 \%$ \\
& $(83.5,100)$ & & $(93.5,100)$ \\
\hline
\end{tabular}

Table 4. Final diagnoses in study patients with or without intrahepatic biliary dilation.

\begin{tabular}{lccccc} 
& \multicolumn{3}{c}{ Obstructive jaundice } & No jaundice & \\
& \multicolumn{2}{c}{ N=51 } & \multicolumn{2}{c}{ N=56 } & \\
& IHID & No IHD & IHI & No IHID & Overall \\
& & & & & \\
Benign (n=91) & & & & & \\
No identifiable obstructive lesion & $4(3.7)$ & $15(14.0)$ & $6(5.6)$ & $28(26.1)$ & $53(49.5)$ \\
Chronic pancreatitis & $2(1.8)$ & $9(8.4)$ & $2(1.8)$ & $14(13.0)$ & $27(25.2)$ \\
CBD stone & $1(0.9)$ & $2(1.8)$ & - & $3(2.8)$ & $6(5.6)$ \\
Ampullary stenosis & - & $1(0.9)$ & $1(0.9)$ & $1(0.9)$ & $3(2.8)$ \\
Ampullary adenoma & - & $1(0.9)$ & - & $1(0.9)$ & $2(1.8)$ \\
Malignant (n=16) & & & & & \\
Adenocarcinoma of pancreas & $1(0.9)$ & $5(4.6)$ & - & - & $6(5.6)$ \\
Cholangiocarcinoma & $2(1.8)$ & $4(3.7)$ & - & - & $6(5.6)$ \\
Other neoplasms & $1(0.9)$ & $3(2.8)$ & - & - & $4(3.7)$ \\
\hline
\end{tabular}

IHD, intrahepatic biliary dilation; CBD, common bile duct.

Table 5. Multivariate regression analysis evaluating the influence of patient age, symptoms, size of common bile duct and presence of intrahepatic biliary dilation on the probability of malignancy in patients with dilated common bile duct and obstructive jaundice.

\begin{tabular}{lccc} 
& Odds Ratio & $95 \%$ Confidence interval & P value \\
Age & 1.08 & $1.00,1.17$ & 0.03 \\
Abdominal pain & 0.40 & $0.03,4.80$ & 0.47 \\
\hline Weight loss & 0.57 & $0.10,3.12$ & 0.51 \\
Diameter of CBD & 0.90 & $0.63,1.30$ & 0.60 \\
\hline Intrahepatic biliary dilation & 0.48 & $0.05,4.51$ & 0.52 \\
\hline
\end{tabular}

$\mathrm{CBD}$, common bile duct. 
er in patients with associated ObJ. EUS-FNA is highly accurate in diagnosing malignancy in this patient subset besides being able to identify other potential etiology for biliary dilation including previously missed CBD stones and chronic pancreatitis.

\section{References}

1. Kim JE, Lee JK, Lee KT, et al. The clinical significance of common bile-duct dilatation in patients without biliary symptoms or causative lesions on ultrasonography. Endoscopy 2001;33:495-500.

2. Malik S, Kaushik N, Khalid A, et al. EUS yield in evaluating biliary dilatation in patients with normal serum liver enzymes. Dig Dis Sci 2007;52:508-12.

3. Songür Y, Temuçin G, Sahin B. Endoscopic ultrasonography in the evaluation of dilated common bile duct. J Clin Gastroenterol 2001;33:302-5.

4. Menges M, Lerch MM, Zeitz M. The double duct sign in patients with malignant and benign pancreatic lesions. Gastrointest Endosc 2000;52:74-7.
5. Dearden JC, Ayaru L, Wong V, van Someren N. The double duct sign. Lancet 2004;364:302.

6. Chawla S, Trick WE, Gilkey S, Attar BM. Does cholecystectomy status influence the common bile duct diameter? A matchedpair analysis. Dig Dis Sci 2010;55:1155-60.

7. Lin F, Staerkel G. Cytologic criteria for well differentiated adenocarcinoma of the pancreas in fine-needle aspiration biopsy specimens. Cancer 2003;99:44-50.

8. Sahai AV, Zimmerman M, Aabakken L, Tarnasky PR, Cunningham JT, van Velse A, et al. Prospective assessment of the ability of endoscopic ultrasound to diagnose, exclude, or establish the severity of chronic pancreatitis found by endoscopic retrograde cholangiopancreatography. Gastrointest Endosc 1998;48:18-25.

9. Catalano MF, Sahai A, Levy M, et al. EUSbased criteria for the diagnosis of chronic pancreatitis: the Rosemont classification. Gastrointest Endosc. 2009;69:1251-61.

10. Dancygier H, Nattermann C. The role of endoscopic ultrasonography in biliary tract disease: obstructive jaundice. Endoscopy 1994;26:800-2.

11. Avni Y, Rattan J, Bar-Meir S. The signifi- cance of unexplained dilated common bile duct at endoscopic retrograde cholangiopancreatography. Endoscopy 1988;20: 292-3.

12. Mueller PR, Ferrucci JT, Simeone JF, et al. Postcholecystectomy bile duct dilatation: myth or reality? AJR Am J Roentgenol 1981;136:355-8.

13. Feng B, Song Q. Does the common bile duct dilate after cholecystectomy? Sonographic evaluation in 234 patients. AJR Am J Roentgenol 1995;165:859-61.

14. Hamilton I, Ruddell WS, Mitchell CJ, et al. Endoscopic retrograde cholangiograms of the normal and post-cholecystectomy biliary tree. Br J Surg 1982;69:343-5.

15. Wallace MB, Hawes RH, Durkalski V, et al. The reliability of EUS for the diagnosis of chronic pancreatitis: interobserver agreement among experienced endosonographers. Gastrointest Endosc 2001;53:294-9.

16. Pungpapong S, Wallace MB, Woodward TA, et al. Accuracy of endoscopic ultrasonography and magnetic resonance cholangiopancreatography for the diagnosis of chronic pancreatitis: a prospective comparison study. J Clin Gastroenterol 2007; 41:88-93. 